(1)

CrossMark

\section{Short-term outcomes of COVID-19 and risk factors for progression}

\author{
To the Editor:
}

Coronavirus disease 2019 (COVID-19) has now become a worldwide health concern. The severity of COVID-19 has been classified as mild, moderate, severe and critical [1]. To date, there have been a few studies focused on the clinical course and outcome of critical cases [2-4]. However, information regarding outcomes of mild-to-moderate cases is lacking, despite the fact that mild-to-moderate cases have accounted for approximately $80 \%$ of laboratory-confirmed patients [1,5]. This study aimed to investigate short-term outcomes of patients rated with different severities on admission, and to identify risk factors for progression, thereby helping the management of COVID-19 in clinical practice.

All consecutive patients with laboratory-confirmed COVID-19 admitted to The First People's Hospital of Jingzhou and to Xiangyang Central Hospital (tertiary hospitals located in Jingzhou City and Xiangyang City, Hubei Province, China) between 16 January and 24 February 2020 were unselectively enrolled. Diagnosis of COVID-19 was made according to the Diagnosis and Treatment Program for COVID-19 (Trial Version 5), released by China's National Health Commission [6], and complied with World Health Organization interim guidance [7]. Mild cases were defined when patients only presented with mild symptoms and did not have a radiographic appearance of pneumonia. Moderate cases were defined when patients presented with fever and/or respiratory symptoms and had a radiographic appearance of pneumonia. Severe cases were defined when patients presented with one of the following: 1) respiratory distress with respiratory rate $\geqslant 30$ breaths $\cdot \min ^{-1} ; 2$ ) finger oxygen saturation $\leqslant 93 \%$ at resting state; 3 ) arterial oxygen tension/inspiratory oxygen fraction $\leqslant 300 \mathrm{mmHg}$. Critical cases were defined when patients presented with one of the following: 1) respiratory failure requiring mechanical ventilation; 2) shock; 3) other organ failure requiring admission to the intensive care unit [6]. Progression was determined once the severity got worse, and the dates were recorded in order to calculate the interval between admission date and progression date. Unchanged severity during the whole observation period, despite having a severe/ critical disease condition on admission, was not classified as progression. Clinical features, laboratory findings and treatments, as well as clinical outcomes (i.e. discharge, the severity of the disease, mortality, length of stay) of patients were recorded and monitored up to 1 March 2020. This study was approved by the ethics commissions of both hospitals (The First People's Hospital of Jingzhou 42016803-T, and Xiangyang Central Hospital 2020-001). Written informed consent was waived due to the emergency of the COVID-19 pandemic.

In total, 301 confirmed cases were enrolled. Of these, 150 (49.8\%) were male and the median (interquartile range (IQR)) age was 51.0 (36.0-63.0) years. On admission, the median (IQR) disease duration was 6.0 (4.0-9.0) days, and the numbers of mild, moderate, severe and critical cases were eight (2.6\%), 245 (81.4\%), $36(12.0 \%)$ and $12(4.0 \%)$, respectively. A standard treatment protocol was applied. Of the mild and moderate patients, $214(84.6 \%)$ were treated only with medical treatment as initial therapy (antiviral therapy combined with as-needed antibiotics, expectorant, Chinese patent medicines, etc.), while 39 (15.4\%) received both medical treatment and low-flow oxygen inhalation. All of the 48 severe and critical patients received both medical treatment and oxygen therapy, including $22(45.8 \%)$ who received low-flow oxygen inhalation, 15 (31.3\%) high-flow inhalation, eight (16.7\%) noninvasive ventilation and three (6.2\%) invasive ventilation.

By the final day of follow-up, the median (IQR) observation time was 24.0 (17.5-30.0) days, and the median (IQR) disease duration of the included patients was 30.0 (23.0-36.0) days. Of the 301 included

@ERSpublications

With a median (IQR) follow-up time of 24.0 (17.5-30.0) days, progression occurred in $19.6 \%$ moderate, $27.8 \%$ severe and $66.7 \%$ critical COVID-19. Neutrophil-to-lymphocyte ratio $\geqslant 2.973$, age $\geqslant 50$ years, male sex and comorbidity were associated with progression. https://bit.ly/3crsJr0

Cite this article as: Long L, Zeng X, Zhang X, et al. Short-term outcomes of COVID-19 and risk factors for progression. Eur Respir J 2020; 55: 2000990 [https://doi.org/10.1183/13993003.00990-2020]. 
patients, $156(51.8 \%)$ patients were treated only with medical treatment, and low-flow oxygen inhalation, high-flow oxygen inhalation, noninvasive ventilation, invasive ventilation and extracorporeal membrane oxygenation were given to $104(34.6 \%), 42(14.0 \%), 17(5.6 \%)$, four $(1.3 \%)$ and one $(0.3 \%)$ patients, respectively (some patients received more than one type of oxygen therapy). None of the mild cases had experienced progression. $48(19.6 \%)$ out of the 245 moderate patients experienced progression during hospitalisation; among them, $14(5.7 \%)$ turned moderate and six (2.5\%) were discharged, while $21(8.6 \%)$ were severe, two $(0.8 \%)$ were critical and five $(2.0 \%)$ died by the end-point. $10(27.8 \%)$ out of the 36 severe cases had experienced progression; among them, one (2.8\%) turned moderate and one (2.8\%) was discharged, while two (5.6\%) were severe, two (5.6\%) were critical and four $(11.1 \%)$ died. As for the 12 critical cases, eight (66.7\%) experienced progression (died). By the final day of follow-up, overall, 66 (21.9\%) patients had experienced progression. The numbers of discharged, mild, moderate, severe, critical and dead patients were $156(51.8 \%)$, five (1.7\%), $84(27.9 \%), 34(11.3 \%)$, five $(1.7 \%)$ and $17(5.6 \%)$, respectively. The median (IQR) time from admission to disease progression was 6.0 (5.0-9.0) days, and from admission to discharge was 22.0 (16.0-25.0) days.

When assessed by receiver operating characteristic curve, the optimal cut-off value of age for differentiating patients with progression and no progression was 50 years (area under the curve 0.7366 , sensitivity $81.8 \%$, specificity $58.3 \%$ ), and the optimal value of the neutrophil-to-lymphocyte ratio (NLR) was 2.973 (area under the curve 0.7338 , sensitivity $75.8 \%$, specificity $66.8 \%$ ). Variables including baseline characteristics, laboratory findings and chest computed tomography scan findings were analysed by univariate Cox regression analyses, and sex, age, comorbidities, NLR, eosinophil count and C-reactive protein level were identified as significant variables, which were then included in multivariate Cox regression analyses. Having an NLR $\geqslant 2.973$ (hazard ratio (HR) 2.641, 95\% CI 1.421-4.908; $\mathrm{p}=0.002$ ), age $\geqslant 50$ years (HR 2.504, 95\% CI 1.202-5.215; $\mathrm{p}=0.014$ ), being male (HR 2.004, 95\% CI 1.101-3.647; $\mathrm{p}=0.023$ ) and having comorbidity (HR 1.969, 95\% CI 1.085-3.571; p=0.026) were identified as risk factors for progression by multivariate Cox regression analyses. Kaplan-Meier curves were used to estimate time-dependent hazards categorised by these risk factors (figure 1).
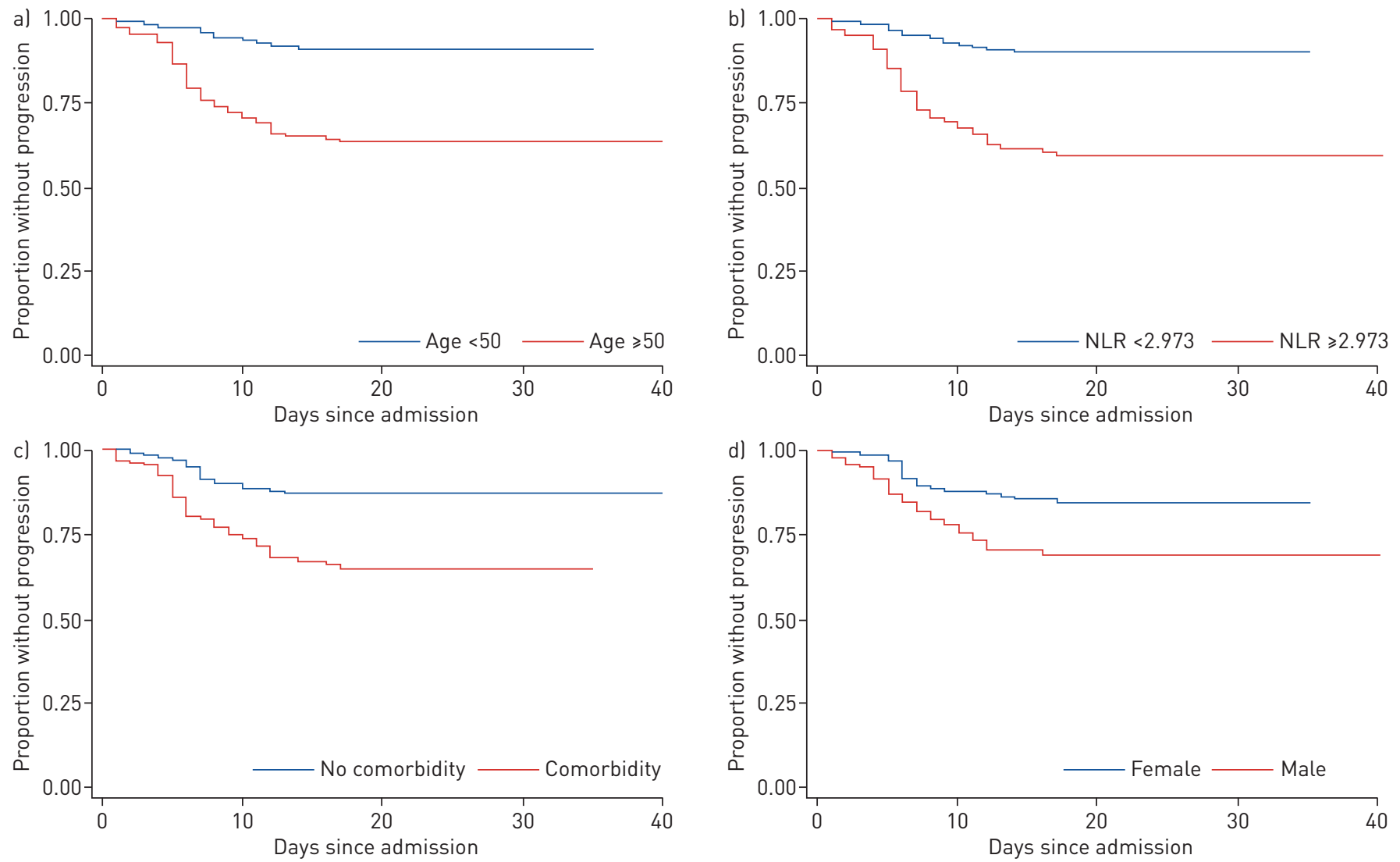

FIGURE 1 Time from hospital admission to progression, categorised by risk factors: al age <50 years versus $\geqslant 50$ years; b) neutrophil-to-lymphocyte ratio (NLR) $<2.973$ versus $\geqslant 2.973$; c) with comorbidity versus without comorbidity; and d) female versus male. 
All patients who experienced progression during hospitalisation had at least one of the risk factors. 54 $(81.8 \%)$ of them were aged $\geqslant 50$ years, $50(75.8 \%)$ had an NLR $\geqslant 2.973,46(69.7 \%)$ had comorbidity and 44 (66.7\%) were male.

As far as we know, this is the first study that focuses on clinical outcomes of COVID-19 patients rated with different severities on admission. Considering that a few of the mild-to-moderate cases developed a critically ill condition, we chose the progression of COVID-19 as the primary outcome rather than a composite end-point (one or more of death, admission to the intensive care unit, or use of mechanical ventilation). We believe the findings from this study will be helpful to prevent progression at an earlier stage for general COVID-19 patients, and could be a supplement to a recent study that reported patients with any comorbidity were more likely to achieve the composite end-point than those without [8].

Our study has some limitations. First, hospitals will have given priority to more severe patients, which might have led to a lower proportion of mild cases in this study. Secondly, although we had a median (IQR) follow-up time of 24.0 (17.5-30.0) days, at the end-point, nearly half of the patients were still hospitalised. Progression could still occur in the patients who did not get worse during our observation period; thus, the actual progression rate might be higher than the results shown in the present study.

In conclusion, with a median follow-up time of 24.0 (17.5-30.0) days, we found that progression occurred in $19.6 \%$ of moderate cases, $27.8 \%$ of severe cases and $66.7 \%$ of critical cases during hospitalisation. An NLR $\geqslant 2.973$, age $\geqslant 50$ years, male sex and comorbidity were associated with progression.

Li Long $\oplus^{1,4}$, Xiansheng Zeng ${ }^{2,4}$, Xu Zhang ${ }^{3,4}$, Wei Xiao ${ }^{3,4}$, E Guo ${ }^{2,4}$, Wenzhi Zhan ${ }^{1}$, Xuejiao Yang ${ }^{2}$, Chunyan $\mathrm{Li}^{3}$, Caiyun $\mathrm{Wu}^{2}$, Tingting $\mathrm{Xu}^{1}$, Chen Zhan ${ }^{1}$, Yuehan Chen ${ }^{1}$, Mei Jiang ${ }^{1}$, Nanshan Zhong ${ }^{1}$ and Kefang Lai ${ }^{1}$

${ }^{1}$ State Key Laboratory of Respiratory Disease, National Clinical Research Center for Respiratory Disease, Guangzhou Institute of Respiratory Health, The First Affiliated Hospital of Guangzhou Medical University, Guangzhou, China.

${ }^{2}$ Xiangyang Central Hospital, Affiliated Hospital of Hubei University of Arts and Science, Xiangyang, China. ${ }^{3}$ The First

People's Hospital of Jingzhou, Jingzhou, China. ${ }^{4}$ These authors contributed equally to this article.

Correspondence: Kefang Lai, State Key Laboratory of Respiratory Disease, National Clinical Research Center for Respiratory Disease, Guangzhou Institute of Respiratory Health, The First Affiliated Hospital of Guangzhou Medical University, 151 Yanjiang Rd, Guangzhou, 510120, China. E-mail: klai@163.com

Received: 6 March 2020 | Accepted after revision: 11 April 2020

Acknowledgements: Our thanks go to all doctors and nurses in The First People's Hospital of Jingzhou, and Xiangyang Central Hospital.

Conflict of interest: None declared.

Support statement: This study was supported by Incubative Project for Innovation Team of Guangzhou Medical University (GMU) (2017-159), and Clinical Research Foundation of GMU (2017-160). The funder of the study had no role in study design, data collection, data analysis, data interpretation, or writing of the report. Funding information for this article has been deposited with the Crossref Funder Registry.

\section{References}

1 WHO-China Joint Mission. Report of the WHO-China Joint Mission on Coronavirus Disease 2019 (COVID-19). www.who.int/docs/default-source/coronaviruse/who-china-joint-mission-on-covid-19-final-report.pdf Date last updated: 24 February 2020. Date last accessed: 4 March 2020.

2 Yang $\mathrm{X}, \mathrm{Yu} \mathrm{Y,} \mathrm{Xu} \mathrm{J,} \mathrm{et} \mathrm{al.} \mathrm{Clinical} \mathrm{course} \mathrm{and} \mathrm{outcomes} \mathrm{of} \mathrm{critically} \mathrm{ill} \mathrm{patients} \mathrm{with} \mathrm{SARS-CoV-2} \mathrm{pneumonia} \mathrm{in}$ Wuhan, China: a single-centered, retrospective, observational study. Lancet Respir Med 2020; 8: 475-481.

3 Huang C, Wang Y, Li X, et al. Clinical features of patients infected with 2019 novel coronavirus in Wuhan, China. Lancet 2020; 395: 497-506.

4 Chen N, Zhou M, Dong X, et al. Epidemiological and clinical characteristics of 99 cases of 2019 novel coronavirus pneumonia in Wuhan, China: a descriptive study. Lancet 2020; 395: 507-513.

5 Wu Z, McGoogan JM. Characteristics of and important lessons from the coronavirus disease 2019 (COVID-19) outbreak in China: summary of a report of 72314 cases from the Chinese Center for Disease Control and Prevention. JAMA 2020; 323: 1239-1242.

6 National Health Commission of the People's Republic of China. New Coronavirus Infection Pneumonia Diagnosis and Treatment Program (Trial Version 5). Available from: www.nhc.gov.cn/yzygj/s7653p/202002/3b09b894ac9b4 204a79db5b8912d4440.shtml Date last updated: 5 February 2020. Date last accessed: 8 February 2020.

7 World Health Organization. Clinical management of severe acute respiratory infection when novel coronavirus $(\mathrm{nCoV})$ infection is suspected. Available from: www.who.int/publications-detail/clinical-management-of-severe-acuterespiratory-infection-when-novel-coronavirus-(ncov)-infection-is-suspected Date last updated: 28 January 2020. Date last accessed: 8 February 2020.

8 Guan WJ, Liang WH, Zhao Y, et al. Comorbidity and its impact on 1590 patients with COVID-19 in China: a nationwide analysis. Eur Respir J 2020; 55: 2000547. 